
temps de la convergence?

\title{
Littératies informationnelles, médiatiques et numériques : de la concurrence à la convergence?
}

Information, Media, and Digital Literacies: From Competition to Convergence

\section{Olivier Le Deuff}

\section{(2) OpenEdition}

Journals

Édition électronique

URL : http://journals.openedition.org/edc/3411

DOI : 10.4000/edc.3411

ISSN : 2101-0366

Éditeur

Université Lille-3

Édition imprimée

Date de publication : 30 juin 2012

Pagination : 131-147

ISBN : 978-2-917562-07-9

ISSN : $1270-6841$

Référence électronique

Olivier Le Deuff, «Littératies informationnelles, médiatiques et numériques : de la concurrence à la convergence? ", Études de communication [En ligne], 38 | 2012, mis en ligne le 30 juin 2014, consulté le 19 avril 2019. URL : http://journals.openedition.org/edc/3411 ; DOI : 10.4000/edc.3411

Ce document a été généré automatiquement le 19 avril 2019

(c) Tous droits réservés 


\title{
Littératies informationnelles, médiatiques et numériques : de la concurrence à la convergence?
}

Information, Media, and Digital Literacies: From Competition to Convergence

\author{
Olivier Le Deuff
}

1 Le constat d'une convergence médiatique liée au numérique succède à une période qui pourrait correspondre à une « querelle » des littératies. Les littératies informationnelles ( information literacy) et médiatiques (media literacy) se sont donc disputées pendant plusieurs années une forme de leadership, chacune prétendant contenir l'autre. En parallèle, la littératie informatique (computer literacy) a connu également des évolutions du fait du numérique au point d'être souvent intégrée au projet d'une littératie du numérique (digital literacy).

2 L'émergence de nouvelles littératies a accru cette complexité et cette dispersion des forces. Les actuelles perspectives offertes par la translittératie (transliteracy) (Thomas, 2007) ou bien encore par la MIL (Media and Information Literacy) permettraient de les regrouper toutes dans un même projet, à condition de réellement parvenir à de réelles avancées sans répéter des étapes précédemment déjà opérées. Nous songeons notamment aux fameux référentiels de compétences en vogue dans les années 80 et 90 et qui apparaissent aujourd'hui insuffisants.

3 De plus, s'ajoute au constat de convergence, la nécessité du développement d'une culture de la participation bien mis en avant par les nouvelles littératies liées à l'émergence du web 2.0 (social literacy, tag literacy) et particulièrement la new media literacy. Cette dernière insiste sur l'intérêt de former les nouvelles générations à tirer profit des nouvelles technologies et donc à davantage y prendre part. Le projet mené notamment par Henry Jenkins (Jenkins, 2008) met fortement l'accent sur les aspects participatifs comme en témoigne le slogan du projet « connect, communicate, create, collaborate ». 
Cependant, un retour sur la littératie apparaît de ce fait opportun. Le concept est importé du monde anglo-saxon avec une transition opérée notamment par les québécois pour l'adapter en langue française. L'usage du terme de littératie implique un lien avec l'écriture et les rapports que la culture entretient avec la raison graphique. Mais il ne faudrait pas voir dans la littératie une vision simplement basée sur l'alphabétisme mais bel et bien sur le concept de texte tel qu'il est défini par Yves Jeanneret ${ }^{1}$. La littératie ainsi définie, le concept apparaît toujours adéquat face aux mutations du numérique. Le numérique ne change donc en rien le contexte de crise associée au concept, ce qu'Harvey Graff (Graff, 1987) avait déjà bien mis en évidence. Il distingue trois grandes tendances qui sont toutes les trois liées historiquement avec la notion de crise :

- Les habiletés essentielles (lire, écrire, compter) et leurs mesures en fonction des résultats des élèves sont sans cesse l'enjeu de discours catastrophistes ou alarmistes et rejoignent la crainte perpétuelle de la baisse de niveau.

- L'étroite relation qu'entretient la littératie avec l'éducation morale et la citoyenneté. Les discours en font un préalable nécessaire à la bonne marche de la société. Actuellement, les discours portent plutôt d'ailleurs sur la bonne adéquation à la «société de l'information ».

- L'émergence incessante et croissante de nouvelles littératies. Ces littératies sont parfois liées à des effets de mode et nullement durables. Graff (Graff, 1985, p. 321) évoquait d'ailleurs déjà la teleliteracy.

5 Cette crise " éducative » se remarque par l'obsession de l'évaluation des compétences et la volonté d'un retour sur investissement rapide qui porte sur les formations à l'information et aux médias. Pourtant, le fait d'envisager un rassemblement des littératies médiatique, informationnelle et informatique nécessite un examen plus profond et des objectifs à long terme.

\section{Méthodologie}

6 Chacune de ces littératies possède sa propre histoire, ses propres acteurs et théoriciens et notre propos n'est pas de les présenter toutes de manière exhaustive. La plupart de ces littératies présente une tentation hégémonique visant à englober les autres. Pourtant, les frontières deviennent de plus en plus floues entre elles. Cet examen repose sur un corpus de près de 300 textes de nature scientifique et pédagogique majoritairement anglo-saxons autour de l'information literacy, de la media literacy et de la digital literacy. D'autres textes sur de nouvelles littératies (social literacy, tag literacy, etc.) ont été également examinés. Nous présentons donc une approche comparatiste pour montrer le cheminement et l'évolution de «territoires » qui sont de moins en moins distincts.

7 Nous utilisons ici également des données issues d'une enquête en ligne sur la culture de l'information $(\mathrm{n}=793)$ auprès de professionnels de l'information francophone.

8 Nous souhaitons ici examiner en quoi et comment la convergence médiatique et numérique peut entrainer une "convergence disciplinaire». Nous entendons par convergence numérique, le regroupement d'outils autrefois distincts (téléphone, ordinateur, téléviseur, baladeur) au sein d'environnements qui tend à les fusionner ou les rendre compatibles que ce soit sur Pc ou sur Smartphones du fait qu'ils traitent les données de façon numérique.

9 Cette convergence réinterroge la relation à la technique des littératies. L'importance d'une culture technique parait être un premier point de convergence à examiner, tant les 
outils, les usages, les formats influent sur la définition même des littératies et des objectifs qui leur sont allouées. Cet objectif de culture technique rejoint celui de l'accès à la majorité de l'entendement qui permet à l'individu de gérer au mieux son environnement informationnel, médiatique et numérique en tant que citoyen "éclairé » (Le Deuff, 2009).

\section{Littératie, un concept résistant et adaptionniste}

La littératie nous parait être un concept adéquat au-delà de son utilisation grandissante au sein des productions francophones. Le concept possède trois sens principaux qui démontrent également ses possibilités d'extension de signification.

- Premier sens : capacité à lire et écrire (a simple ability to read and write).

- Deuxième sens: Possession de compétence et d'habileté (Having some skill and competence).

- Troisième sens : Élément d'apprentissage (Element of learning).

11 Nous rattachons également le concept à celui de texte d'Yves Jeanneret pour signifier qu'il possède une résistance et une pertinence qui demeure au gré de l'évolution des médias et ce afin d'éviter un discours révolutionnaire ou de rupture.

Cette résistance est aussi due à sa plasticité ou à ce que la chercheuse Tracy Whalen (Whalen, 2004, p.16) nomme son élasticité, c'est-à-dire sa capacité à évoluer et à s'adapter selon les contextes, qu'ils soient techniques ou nationaux.

13 Le contexte économique influe fortement les littératies informationnelles et médiatiques et leur manière de voir. L'information literacy est très marquée par une vision utilitariste depuis la création du terme par Zurkowski ${ }^{2}$ en 1974. Son développement au sein des sphères bibliothéconomiques notamment américaines s'inscrit dans un mouvement similaire en tentant de démontrer l'utilité économique des bibliothèques. Par conséquent, les visions sont celles de formations courtes et essentiellement pratiques pouvant être réinvesties immédiatement. L'objectif étant d'adosser à la prétendue «société de l'information" dont la valeur marchande de base serait l'information, une formation permettant que les travailleurs soient préparés à valoriser au mieux cette nouvelle matière première. La media literacy demeure aussi dans une sorte de réponse à des impératifs économiques. Toutefois, il s'agit plutôt de donner des moyens de réponse face à la multiplication des écrans et notamment face aux stratégies publicitaires.

Le concept media literacy n'a pas de réel équivalent francophone si ce n'est la traduction « littératie médiatique » employée par les québécois. En effet, le concept éducation aux médias ne recouvre pas totalement les mêmes objectifs et la distinction entre media literacy et media education est parfois effectuée. Une définition avait ainsi émergé aux États-Unis en 1992 lors de la réunion à Aspen (Aspen Institute Report of the National Leadership Conference on Media Literacy) qui avait réuni plus d'une trentaine de spécialistes du domaine :

« La littératie médiatique est la capacité à accéder, analyser, évaluer et à créer des medias dans une variété de formes $»^{3}$.

15 Au niveau européen, elle s'est donc vue accordée une légitimité mais qui apparaît fortement marginale en étant une concession au sein de l'agenda numérique et s'inscrit dans l'initiative «i2010 - Une société de l'information pour la croissance et l'emploi ». Divina Frau-Meigs décrit ce contexte difficile : 
L'éducation aux médias apparaît comme un instrument au profit des politiques de déréglementation: elle semble être une concession des forces néo-libérales pour pallier toutes les conquêtes de la dérégulation (assouplissement des quotas, autorisation du placement de produit, soutien des nouvelles formes de publicité, encouragement de l'auto-régulation...). C'est en quelque sorte le bonbon qui fait passer l'amertume de la pilule (Frau-Meigs, 2011).

La digital literacy ne déroge pas à la situation des deux autres en prenant peu à peu le dessus sur une computer literacy d'essence davantage informatique et basée sur de la programmation. Elle préconise surtout une vision pratique et basée sur des usages qui abandonne souvent toute compréhension un peu plus poussée. Bawden (Bawden, 2008) montre bien déjà un début de convergence dans ses perspectives et la définition ${ }^{4} \mathrm{du}$ créateur du terme Paul Gilster (Gilster, 1998) montrait déjà en 1998 toute son amplitude potentielle.

Les littératies médiatiques, informationnelles et numériques apparaissent donc clairement "adaptionnistes », tant il s'agit de faire face ou de répondre à des impératifs économiques ou pragmatiques vis-à-vis de technologies en émergence. Le peu de place accordé dans les programmes et les cursus n'a fait que renforcer ce désir de prouver leur utilité comme en démontre la régulière volonté d'évaluer le retour sur investissement particulièrement pour l'information literacy5.

\section{La querelle des littératies et l'émergence de nouvelles littératies}

La question du leadership entre les trois littératies n’a jamais été vraiment tranchée. Chacune considérant les autres comme un sous-ensemble. Il existe des positions aussi historiques voire épistémologiques qui demeurent en suspens notamment vis-à-vis des environnements web: est-ce que ce sont des médias, de l'information, ou des supports numériques? La question est fréquemment posée d'ailleurs en ce qui concerne le moteur de recherche Google dont les frontières deviennent peu évidentes d'autant que tout finit par tomber dans son « entonnoir » (Gallezot et Simmonnot, 2009).

19 Les marques d'expression de «leadership » ou de démonstration de "supériorité » se perçoivent dans les illustrations qui démontrent déjà des formes de convergence mais en laissant toujours un primat dans la manière de voir comme le montre l'illustration cidessous (Figure 1 : la fleur de l'information literacy). 


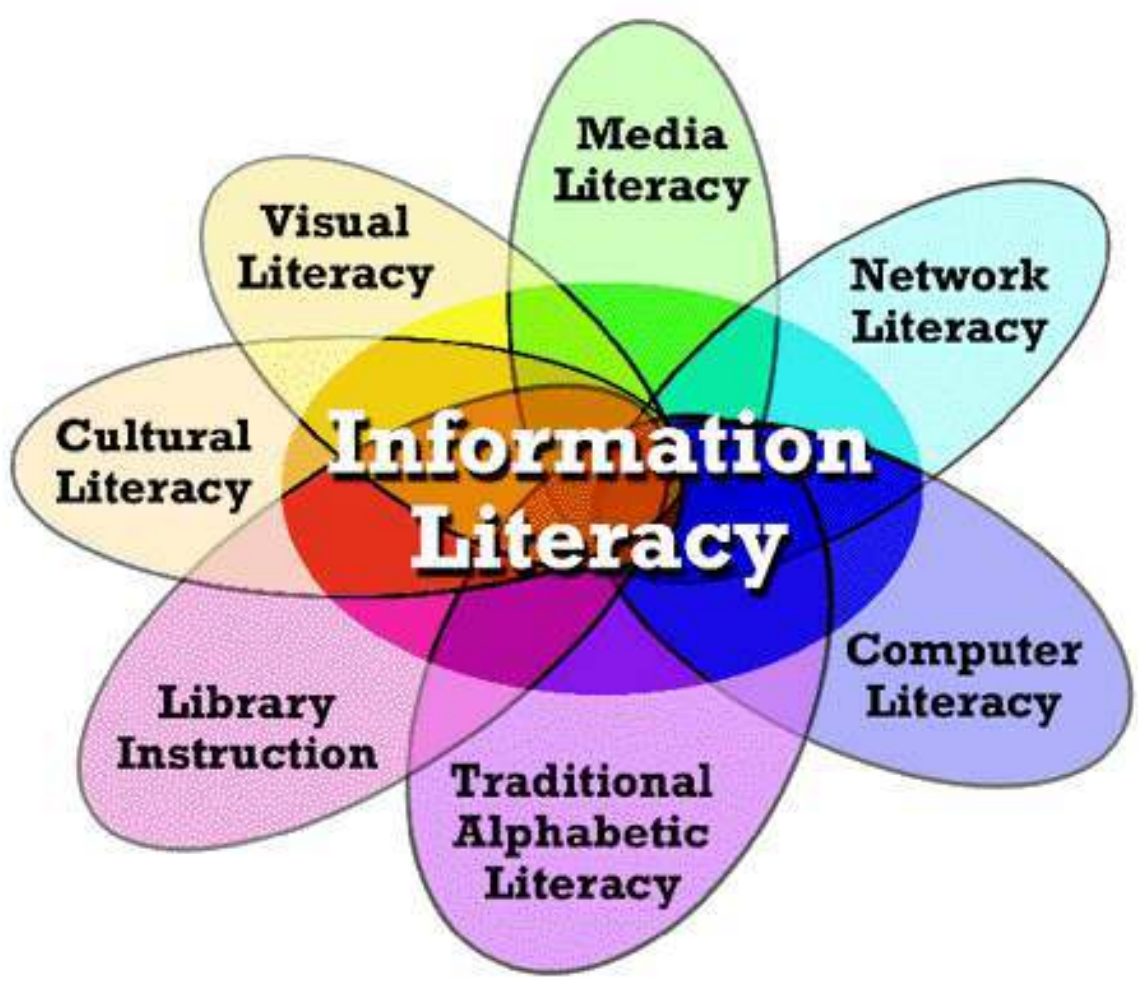

Figure 1 : La fleur de l'information literacy ${ }^{6}$ sans cesse source de nouvelles littératies, certaines probablement éphémères, mais qui mettent à jour de nouvelles pratiques et usages ainsi que de nouveaux besoins de formation. Il reste que cette «floraison » de nouvelles littératies ne fait qu'accentuer la confusion et la dispersion des moyens.

Bawden (Bawden, 2001 avait ainsi dénombré six littératies autour du concept de digital literacy. Nous avons complété et élargi ce recensement dans le tableau ci-dessous (tableau 1) notamment en faisant figurer de nouvelles littératies liées notamment au web 2.0. Nous pouvons distinguer particulièrement la tag literacy autour du bon usage des dispositifs qui utilisent les folksonomies.

\begin{tabular}{|l|l|l|}
\hline Literacy & $\begin{array}{l}\text { Termes proches } \\
\text { ou dérivés }\end{array}$ & Traduction possible \\
\hline $\begin{array}{l}\text { Information } \\
\text { literacy }\end{array}$ & $\begin{array}{l}\text { Informacy } \\
\text { Infoliteracy }\end{array}$ & $\begin{array}{l}\text { Maîtrise de l'information } \\
\text { Culture informationnelle } \\
\text { Education à l'information }\end{array}$ \\
\hline $\begin{array}{l}\text { Computer } \\
\text { literacy }\end{array}$ & $\begin{array}{l}\text { IT/information technology/electronic/electronic } \\
\text { information literacy }\end{array}$ & $\begin{array}{l}\text { Maîtrise des technologies } \\
\text { informatiques. } \\
\text { Culture informatique }\end{array}$ \\
\hline Critical literacy & Critical thinking & $\begin{array}{l}\text { Education critique } \\
\text { Esprit critique }\end{array}$ \\
\hline
\end{tabular}




\begin{tabular}{|c|c|c|}
\hline Library literacy & Library/bibliographic instruction & $\begin{array}{l}\text { Formation à la recherche en } \\
\text { bibliothèque }\end{array}$ \\
\hline Media literacy & New media literacy & Education aux médias \\
\hline Network literacy & Internet literacy, hyper-literacy, bit literacy & $\begin{array}{l}\text { Maîtrise des réseaux } \\
\text { Culture des réseaux }\end{array}$ \\
\hline Digital literacy & Digital information literacy /e-literacy8 & $\begin{array}{l}\text { Culture numérique } \\
\text { Cyberculture }\end{array}$ \\
\hline Visual literacy & & $\begin{array}{l}\text { Culture visuelle } \\
\text { Éducation à l'image }\end{array}$ \\
\hline Social literacy & $\begin{array}{l}\text { Participatory media literacy } \\
\text { Participation literacy } \\
\text { Tag literacy }\end{array}$ & $\begin{array}{l}\text { Littératie de participation (aux } \\
\text { medias) }\end{array}$ \\
\hline Transliteracy & $\begin{array}{l}\text { MIL } \\
\text { (Media and } \\
\text { Information Literacy) }\end{array}$ & $\begin{array}{l}\text { Translittératie } \\
\text { Culture de l'information et des } \\
\text { médias } \\
\text { Littératie des médias de } \\
\text { l'information }\end{array}$ \\
\hline
\end{tabular}

Tableau 1 : Les littératies voisines de l'information literacy des enviriques l'intérêt qu'il ya à former particulièrement nouvelles générations pour leur en faire tirer la quintessence. Le projet est dirigé par le scientifique et blogueur ${ }^{8}$ Henry Jenkins, directeur du programme des études des médias comparés à l'université du Massachussets (MIT) mais aussi auteur d'un ouvrage sur la convergence (Jenkins, 2006). Ce dernier rappelle la nécessité de dépasser l'illusion de voir les jeunes générations comme des natifs du numériques et qu'il convient de les former à se « connecter, communiquer, créer et collaborer ». Les nouvelles littératies mettent de plus en plus en avant des habiletés autant communicationnelles qu'informationnelles. Il s'agit donc autant de sélectionner l'information que de savoir la produire ou la diffuser.

Toutefois le risque serait de privilégier les capacités communicationnelles de base avec les outils de messagerie voire de développer les bonnes pratiques sur les réseaux sociaux au détriment des compétences informationnelles. Les professionnels de l'information et notamment les bibliothécaires ont permis d'éviter cet oubli lors de la mise en place des tests de mesure de niveau des compétences numériques des étudiants devant entrer en première année dans les universités américaines. En effet, le test produit par la société ETS, Iskills ${ }^{9}$ a fortement évolué du fait de l'influence de bibliothécaires qui sont parvenus à introduire l'évaluation de capacités informationnelles. Le test est d'ailleurs reconnu compatible avec les standards en information literacy de l'ACRL (Association of Colleges \& Research Libraries). L'entreprise américaine évoque d'ailleurs une ICT literacy 
désignant ainsi une littératie des technologies de l'information et de la communication. Le terme étant régulièrement utilisé aussi dans les documents de l'Union Européenne.

Néanmoins, malgré cette prise de conscience d'une formation liées aux nouveaux environnements, l'enjeu est de mettre en place un enseignement qui ne contente pas d'usages simplistes mais qui veille à montrer les présupposés et les fondements des différents outils et médias.

\section{L'importance d'une culture technique}

La somme des outils proposée par les environnements numériques incite à poser la question de la relation des littératies avec la technique. Cette relation n'est pas nouvelle et existait déjà auparavant dans le domaine analogique et au sein de la «raison graphique ». Elle était simplement négligée comme l'ont montré notamment les travaux du philosophe Bernard Stiegler ${ }^{10}$. Il convient donc de replacer les outils du numérique dans la lignée des outils de traitement de la pensée. La littératie est d'essence technique tant il s'agit de manipuler toute une série d'objets qui permettent notamment de traiter et d'organiser l'information. Dans le cas d'une convergence vers un omnimédia (Kernéis et al., 2011 dans ce numéro) dont les usagers et notamment les plus jeunes ne distinguent plus les anciennes frontières, l'importance de développer une culture technique provient de la nécessité de différencier les différents types de contenu mais également de comprendre les mécanismes et les objets techniques qui permettent leur diffusion. La convergence étant d'essence technique, il apparait opportun que la formation le soit également en recherchant le développement d'une "culture technique ». Le philosophe Gilbert Simondon explique son intérêt :

«Il est nécessaire que l'objet technique soit connu en lui-même pour que la relation de l'homme à la machine devienne stable et valide : d'où la nécessité d'une culture technique $»^{11}$.

C'est aussi une manière de rappeler l'importance de la technique dans la constitution de la pensée et de la culture. Quelque part, la diversité des outils employés au sein des environnements numériques constitue une opportunité de changer la relation hiérarchique entre culture et technique afin de sortir de cet héritage mutilé (Perriault, 1998).

27 Cette culture est aussi celle du libre-accès, c'est-à-dire d'environnements qui puissent être examinés du fait de leur ouverture. Le britannique Doug Belshaw, ancien employé de Google qui étudie les littératies numériques, recommande de travailler et de former autour des outils open source et préconise même dans ce cadre le développement d'une " openness literacy $»^{12}$ ce qui implique également de savoir quelque peu « programmer $»^{13}$.

\section{Translittératie : convergences des formations ?}

28 Le récent développement de la translittératie correspond pleinement à ce constat de convergence et à la nécessité de réunir les forces pour tenter de mettre en place une formation qui ne s'effectue plus à la marge. La translittératie répond ainsi à l'appel au rassemblement effectué par Alexandre Serres :

Il faut donc prendre toute la mesure de la numérisation et de la généralisation d'Internet et des TIC à toutes les sphères de la réalité et repenser, à partir de là, les «litéracies» informationnelles. Autrement dit, il faudrait faire une «révolution 
copernicienne » dans la conception et la définition des différentes formations à l'information: partir de la réalité des pratiques et des techniques de l'information, des enjeux qui leur sont liés et bâtir ensuite une culture informationnelle globale, intégrant toutes les dimensions de l'information, notamment ces trois cultures spécifiques, portant d'une part sur les médias, la documentation et les bibliothèques, d'autre part sur l'informatique et les outils. Il faudrait y intégrer l'éducation aux images, plus que jamais nécessaire à l'heure de l'explosion des documents vidéos. Il resterait à y ajouter la « cinquième dimension », essentielle [... ] : la dimension communicationnelle ${ }^{14}$.

La transliterattie n'est pas très éloignée finalement de la vision initiale de la digital literacy proposée par Paul Gilster en 1998 si on examine sa définition : «l'habileté à lire, écrire et interagir par le biais d'une variété de plateformes, d'outils et de moyens de communication, de l'iconographie à l'oralité en passant par l'écriture manuscrite, l'édition, la télé, la radio et le cinéma, jusqu'aux réseaux sociaux ${ }^{15}$. La translittératie opte pour une vision gigogne des littératies et donc également surplombante: Transliteracy does not replace, but rather contains, " media literacy » and also " digital literacy $»^{16}$, ce que montre également la figure ci-dessous.

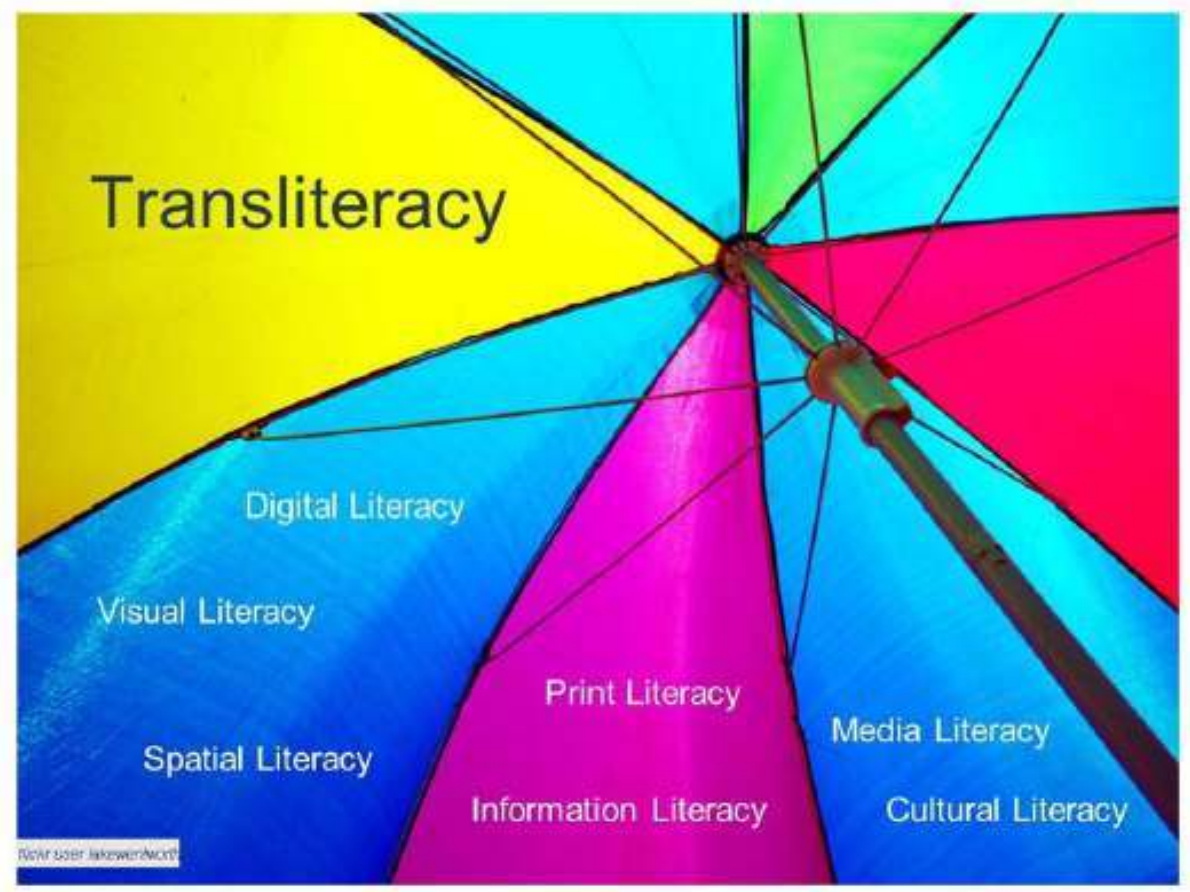

Figure 3 : Le parapluie de la translittératie ${ }^{17}$

Le rassemblement s'observe également au sein de l'UNESCO avec des travaux et des indicateurs pour mesurer la MIL (Media and information literacy) qui s'inscrit dans la continuité des recherches précédentes menées que ce soit en éducation aux médias et dans la formation à l'information. Cette nouvelle littératie de convergence repose sur un triptyque concis qui vise l'efficacité car il s'agit «d'accéder, puis d'évaluer et de comprendre et enfin d'utiliser les médias et l'information dans une série de contextes différents ${ }^{18}$. 


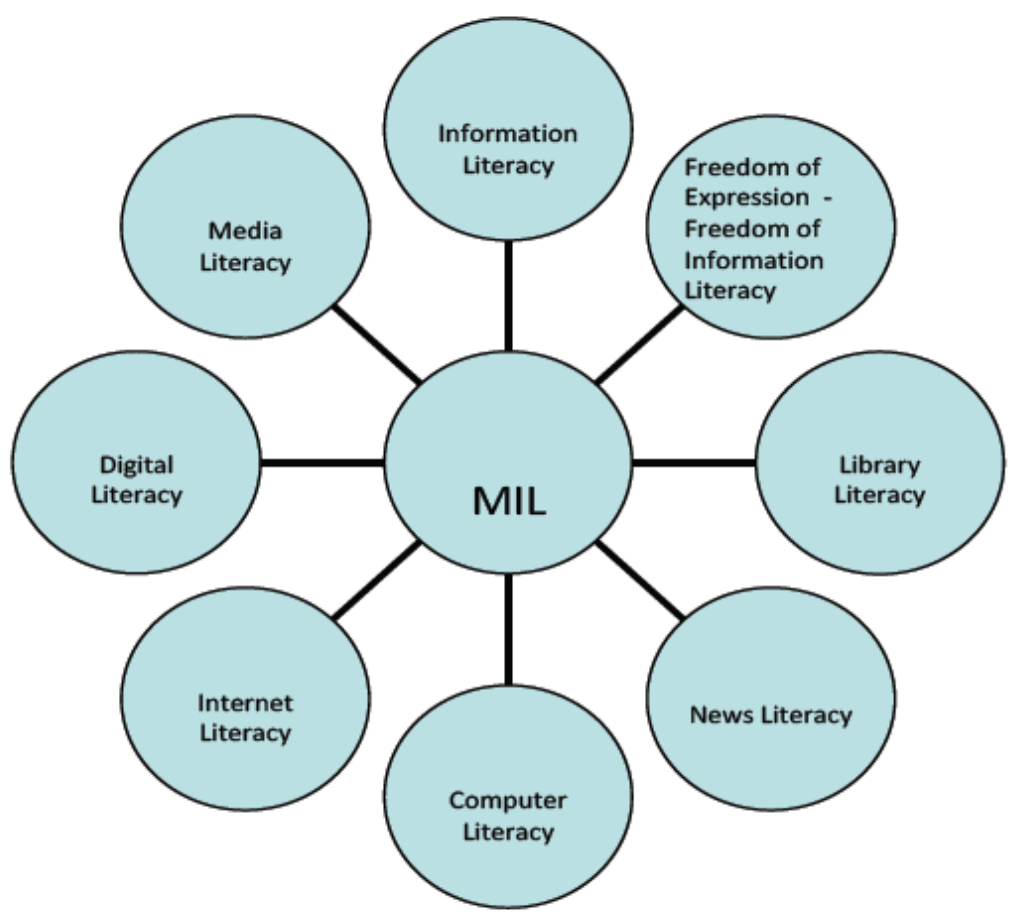

FIgURE 4 : LA MIL ET LES AUTRES LITTÉRATIES ${ }^{19}$

Il reste que ce rassemblement oublie peut-être une littératie que distingue notamment Howard Rheingold: Attention literacy, la littératie de l'attention. La question au-delà du rassemblement des forces pour mettre en place des formations de qualité est bien celle de celle de la capacité à se concentrer durablement sur un support, notamment complexe pour faire face à d'éventuels obstacles cognitifs. Il s'agit de privilégier une attention soutenue et réfléchie face à la facilité et à la tendance à négliger (Le Deuff, 2010) le document pour des usages plus ludiques. En effet, il est difficile de développer l'évaluation de l'information sans un minimum d'attention. Si bien qu'il est possible de distinguer quatre pôles de bases sur lesquels mettre en place une formation commune : l'attention, l'évaluation de l'information, la participation et l'accès libre.

\section{Conclusion : Un concept à discipliner ou disciplinariser?}

Au-delà du flou qui demeure sur les frontières entre les diverses littératies, la convergence numérique entraîne une nécessité de convergence en matière de formation. Désormais, le problème se pose de la création d'une éventuelle discipline et notamment une discipline scolaire qui fasse l'objet d'un enseignement dédié dans les cursus. L'alliance des littératies peut faciliter cette perspective et semble faire l'unanimité comme le montre le résultat de l'enquête en ligne auprès de ceux qui s'accordaient sur la nécessité d'un enseignement dédié dans le secondaire. 


\begin{tabular}{|l|l|l|}
\hline $\begin{array}{l}\text { Pour les personnes favorables à un enseignement spécifique dans le secondaire en information- } \\
\text { documentation, cet enseignement doit-il être }\end{array}$ & Pourcentage \\
\hline Réponse & Décompte & $42.50 \%$ \\
\hline $\begin{array}{l}\text { Couplé avec l'éducation aux médias } \\
\text { et avec l'informatique }\end{array}$ & 337 & $34.43 \%$ \\
\hline Sans réponse & 273 & $11.85 \%$ \\
\hline Couplé avec l'éducation aux médias & 94 & $11.22 \%$ \\
\hline $\begin{array}{l}\text { Propre à l'information- } \\
\text { documentation }\end{array}$ & 89 & \\
\hline
\end{tabular}

\section{TABLEAU 1 : L'ENSEIgNEMENT DOIT-IL ÊTRE SPÉCIfIQUE OU COUPLÉ ?}

Toutefois, il reste à en définir clairement les contenus et les objectifs. Un chantier didactique a amorcé d'autant plus rapidement que les besoins se font sentir et les conséquences de l'absence de formation en la matière sont de plus en plus déplorés. Harvey Graff avait remarqué la littératie s'accompagnait régulièrement de représentations liées à la crise notamment éducative déjà bien évoquée par Hannah Arendt en $1960^{20}$. Les enjeux actuels confirment nettement cette tendance.

Cependant la question n'est plus celle de l'enjeu ou de l'obsession de vouloir mesurer à tout prix les compétences, mais plutôt celle du développement didactique désormais. Les besoins de formation étant urgents du fait des constats chiffrés qu'il n'existe pas de digital natives $^{21}$, il est probable qu'il faille constituer cette nouvelle discipline avec des allers retours incessants entre le terrain et les chercheurs. En effet, il apparait encore difficile de stabiliser pleinement une telle discipline avec des programmes clairement balisés.

\section{BIBLIOGRAPHIE}

Bawden D., (2001), Information and digital literacies : A review of concepts, Journal of Documentation, 57(2), pp. 218-259.

Bawden D., (2008), Origins and concepts of digital literacy, in Lankshear C. \& Knobel M. (eds.), Digital Literacies : concepts, policies and practices (pp. 17-32), New-York, NY, Peter Lang.

Jenkins H. et al., (2008), Confronting the Challenges of Participatory Culture : Media Education for the 21

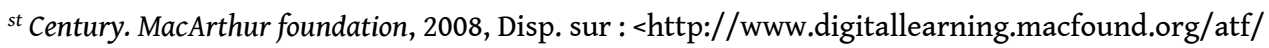
cf/\%7B7E45C7E0-A3E0-4B89-AC9C-E807E1B0AE4E\%7D/JENKINS_WHITE_PAPER.PDF>.

Jenkins H., (2006), Convergence culture : where old and new media collide, New-York, NY University Press. 
Frau-Meigs D., (2011), L'éducation aux médias est-elle nécessaire? A quelles conditions?, Dossiers de l'audiovisuel, janvier 2011.

Gilster P., (1998), Digital literacy.

Graff H. J., (1985), The Labyrinths of Literacy : Reflections on Literacy Past and Present.

Graff H. J., (1987), The Legacies of Literacy: Continuities and Contradictions in Western Culture and Society, Indiana University Press.

Kernéis J. et al., (2012), Convergence médiatique : l'appropriation des médias sociaux par les jeunes utilisateurs. Constats nuancés et pistes de réflexion pour les éducateurs, Étude de communication $n^{\circ} 38$ «L'éducation à l'Information, aux TIC et aux Médias : le temps de la convergence?».

Le Deuff O., (2009), « La culture de l'information et la dimension citoyenne », Les Cahiers du Numérique. « La culture informationnelle », Paris Hermes-Lavoisier, vol. 5, n 3, pp. 39-49.

Le Deuff 0., (2010), «La skholé face aux négligences : former les jeunes générations à l'attention », Communication \& Langages $n^{\circ} 163$, mars 2010, pp. 47-61.

Perriault J., (1998), « Culture technique » Éléments pour l'histoire d'une décennie singulière 1975-1985, in Les cahiers de médiologie, n 6, Gallimard, 1998, pp. 197-214.

Simondon G., (1989), Du mode d'existence des objets techniques, Paris, Aubier.

Simmonnot B. et Gallezot G. (sous la dir. De), (2009), L'entonnoir, Google sous la loupe des sciences de l'information, CF éditions.

Thomas S. et al., (2007), « Transliteracy : Crossing divides » First Monday, volume 12, Number 12 3 December 2007, Disp. sur : <http://firstmonday.org/htbin/cgiwrap/bin/ojs/index.php/fm/ article/viewArticle/2060/1908>.

Whalen T., (2004), « High Stakes, Mistakes, and Staking Claims : Taking a Look at Literacy/ Grosses mises, méprises, mainmises : Regard sur la littératie », Ethnologies, vol. 26, n¹, 2004, pp. 5-34.

\section{NOTES}

1. «Nous pouvons considérer comme des textes une affiche et le jeu qu'elle établit entre images et mots écrits, l'organisation de l'écran d'accueil de notre ordinateur [...], le découpage reconnaissable d'un journal télévisé. Parle de texte, c'est simplement indiquer qu'une forme générale doit organiser un espace d'expression pour qu'il soit lisible, que les messages ne nous parviennent que sous une forme matérielle, concrète, organisée. A cet égard, on peut dire que le texte est toujours un objet technique, mais d'une nature particulière: un objet technosémiotique ", in Yves Jeanneret, Y a-t-il (vraiment) des technologies de l'information ?, PU du Septentrion, 2007, p. 106.

2. Paul G.Zurkowski, The Information Environment: Relationships and Priorities, National Commission on Libraries and Information Science, Washington DC, 1974.

3. Citation originale: «Media Literacy is the ability to access, analyze, evaluate and create media in a variety of forms $»$.

4. «Digital Literacy is the set of attitudes, understanding and skills to handle and communicate information and knowledge effectively, in a variety of media and formats $"$.

5. C'était la thématique de la section «information literacy " en 2008 au $74^{\mathrm{e}}$ congrès de l'IFLA (Internation Federation of Library Association). L'appel à communication mentionnait la nécessité d'évaluer: «Du lycée à l'université, la tendance actuelle indique un nombre d'études 
limité mais en développement sur l'évaluation des étudiants et des formations. En effet, la question de l'évaluation est cruciale pour vérifier l'acquisition des compétences des apprenants et l'efficacité des enseignements".

6. Cette image est reprise régulièrement sur les sites concernant l'information literacy. Notamment ici pour une bibliothéque: < http://www.otis.edu/life_otis/library/ information_literacy/information_competency.html>.

7. Henry Jenkins et al., Confronting the Challenges of Participatory Culture : Media Education for the $21^{\text {st }}$ Century. MacArthur foundation, 2008: <http://www.digitallearning.macfound.org/atf/ cf/\%7B7E45C7E0-A3E0-4B89-AC9C-E807E1B0AE4E\%7D/JENKINS_WHITE_PAPER.PDF>.

8. Confessions of an Aca-fan: the official weblog of Henry Jenkins: <http:// www.henryjenkins.org/index.html>.

9. About Iskills : < http://www.ets.org/iskills/about>.

10. «La culture n'est rien d'autre que la capacité d'hériter collectivement de l'expérience de nos ancêtres et cela a été compris depuis longtemps. Ce qui a été moins compris, c'est que la technique [...] est la condition d'une telle transmission", Bernard Stiegler, "Leroi-Gourhan: l'inorganique organisé », Les Cahiers de médiologie, n 6, pp. 189-193.

11. Gilbert Simondon, Du mode d'existence des objets techniques, Aubier, 1989, p. 32.

12. Doug Belshaw. Openness is literacy, Billet du 29 Août $2011:<$ http://dmlcentral.net/blog/ doug-belshaw/what-do-google-open-source-software-and-digital-literacies-have-common>.

13. Le théoricien des "digital natives », Marc Prensky évoquait d'ailleurs l'intérêt de développer une " programming literacy » démontrant par la même occasion que la culture informatique des jeunes générations n'était pas vraiment innée. Marc Prensky. Programming is the new literacy, in Edutopia.org, Mars 2008 : < http://www.edutopia.org/literacy-computer-programming>.

14. Alexandre Serres, «Tentative de comparaison des trois cultures : culture des médias, culture de l'information, culture des TIC, Document annexe ", Séminaire du GRCDI, Rennes, 14 septembre 2007, URFIST de Rennes, 2008, Disp. sur: <http://www.uhb.fr/urfist/files/ SeminaireGRCDI_2007_A.Serres_TerritoiresCultInfo_TableauComparaison.doc>.

15. La traduction en français a été trouvée sur le blog de François Guite, in Guitef, Disp. sur : $<\mathrm{http}$ ///www.opossum.ca/guitef/archives/003901.html>. Citation originale: "Transliteracy is the ability to read, write and interact across a range of platforms, tools and media from signing and orality through handwriting, print, TV, radio and film, to digital social networks ».

16. Ibid.

17. Image disponible dans le billet de Lassana Magassa du 15 juillet 2011, «Transliteracy: Information Literacy by Another Name?»: < http://www.lassanamagassa.com/2011/07/ transliteracy-information-literacy-by-another-name/>.

18. Citation originale, p. 21 : The ability to 1) access, 2) evaluate/understand and 3) use media and information in a variety of contexts.

19. Source de la figure, Unesco, Media and Information literacy for teachers : <http:// www.unesco.org/new/fileadmin/MULTIMEDIA/HQ/CI/CI/pdf/wmn/curriculum\% 20teachers.pdf>.

20. «La crise générale qui s'est abattue sur tout le monde moderne et qui atteint presque toutes les branches de l'activité humaine se manifeste différemment suivant les pays, touchant des domaines différents et revêtant des formes différentes. En Amérique, un de ses aspects les plus caractéristiques et les plus révélateurs est la crise périodique de l'éducation qui, au moins pendant ces dix dernières années, est devenue un problème politique de première grandeur dont les journaux parlent presque chaque jour [...]. C'est que le problème ici ne se limite sûrement pas à l'épineuse question de savoir pourquoi le petit John ne sait pas lire ", Hannah Arendt, La crise de la culture, Paris, Gallimard, 1989, pp. 223-224.

21. Les résultats du projet ERIAL (Ethnographic Research in Illinois Academic Libraries) démontrent que les étudiants ignorent beaucoup d'éléments notamment en matière de 
recherche d'informations et que les « digital natives » sont bien un mythe. Les premiers éléments et résultats sont disponibles dans cet article : What students don't know. Inside Higher Education, Billet $\quad \mathrm{du} \quad 22$ août 2001: <http://www.insidehighered.com/news/2011/08/22/ erial_study_of_student_research_habits_at_illinois_university_libraries_reveals_alarmingly_poor_information_literacy_and_skills>.

\section{RÉSUMÉS}

Le but de cet article est de montrer comment les littératies informationnelles, médiatiques, numériques sont en train de passer d'une situation de concurrence à une perspective de convergence en matière de formation. Un retour sur le concept de littératie est effectué et l'importance d'une culture technique y est développée. La translittératie et la MIL (Media and Information literacy) y sont présentées comme des perspectives possibles d'une convergence de formation.

The aim of this paper is to show how information literacy, media literacy, and digital literacy are moving from a situation of competition to a desire to converge in training. Starting with a review of the concept of literacy and its appropriateness, the paper then explains why a technical culture is important. Transliteracy and media and information literacy (MIL) are presented as potential perspectives for convergence in training.

\section{INDEX}

Mots-clés : littératie, translittératie, culture technique, convergence numérique, convergence des littératies

Keywords : literacy, transliteracy, digital convergence, technical culture, media and information literacy

\section{AUTEUR}

\section{OLIVIER LE DEUFF}

MICA - Université de Bordeaux 3 